\title{
Organisation de l'intervention en cas d'accident à caractère radiologique sur la base des sous-marins nucléaires lanceurs d'engins de l'île Longue
}

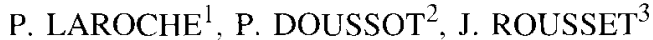

(Manuscrit reçu le 29 juillet 2002, accepté le 4 février 2003)

RÉSUMÉ Dès la survenue d'un accident à caractère radiologique, les intervenants doivent rapidement mener les actions nécessaires à la limitation des conséquences de cet accident sur le personnel, les installations et l'environnement. La phase initiale, qui débute par l'application des fiches réflexes, permet la mise en place de toute l'organisation de commandement et de secours et amène les intervenants sur les lieux de l'accident. L'intervention revêt immédiatement trois aspects : le secours aux blessés, la lutte contre les sinistres et le soutien technique au responsable de mise en oeuvre de l'installation. La mixité des intervenants, à tous les échelons, permet d'assurer simultanément ces missions. Selon qu'il est blessé ou valide, le personnel contaminé est pris en charge dans un chaîne spécifique. La priorité va naturellement vers le secours aux blessés qui sont traités au centre de traitement pour blessés radiocontaminés/Éléments techniques modulaires de l'île Longue (CTBRC/ETM). Le personnel valide de la zone concernée par l'accident a rejoint, dès l'alarme et par action réflexe, les bâtiments refuge. Réputé contaminé, il est pris en charge dès que possible par le personnel du poste de commandement avancé, enregistré et équipé à cet endroit puis évacué vers une structure tampon d'où il sera dirigé vers un des centres de tri et de décontamination sommaire de grande capacité, où il sera décontaminé et contrôlé dans l'attente de son évacuation vers l'extérieur. Une partie est prise en charge pour traitement de la contamination interne ou pour une décontamination fine au CTBRC/ETM.

ABSTRACT Organization of intervention in case of a nuclear accident on the île Longue nuclear submarine base.

When a nuclear accident has occurred, intervention teams have to work out the actions in order to limit results of accident on personnel, installations and environment. Initial stage, that begin applying special cards, allows to organize command and rescue, and brings intervention teams on the accident site. Intervention is composed of three stages: victims' rescue, struggle against conflagration, and technical support to the damaged structure. The diversity of teams allows to carry out these operations at the same time. According as personnel is injured or able bodied, decontamination is carried out in specific structure. Victims' rescue is a priority. Casualties are treated in the Ile Longue treatment center/Technical shelters (CTBRC/ETM). Able-bodied people in the area of accident

\footnotetext{
'Médecin chef de la base opérationnelle de l'île Longue, B.P. 400. 29240 Brest Naval, France.

2 Base operationnelle de l'Île Longue, B.P. 400, 29240 Brest Naval, France.

3 Hôpital d'instruction de armées Clermont Tonnerre, rue colonel Fonferrier, B.P. 41, Brest Naval, France.
} 
have to reach refuges immediately after the alarm. They are presumed contamined and first are checked in the advanced command station. Then they are evacuated, after a stage station, to the large capacity decontamination and triage center, where treatment and control can be effectuated; the evacuation is now possible. Some of them are treated in the île Longue contamination treatment center in case of internal or obstinate contamination.

\section{Introduction}

Le concept de défense en profondeur pour les installations nucléaires précise que toutes les mesures doivent être prises dans le domaine de la sécurité nucléaire afin d'éviter tout accident. Quelles que soient les mesures de prévention mises en œuvre, il exige aussi que le responsable d'une installation, ou d'un ensemble d'installations, mette en place les moyens nécessaires à la gestion d'un accident à caractère radiologique si, bien que peu probable, celui-ci advenait.

Le site de l'Île Longue accueille deux installations nucléaires de base secrète présentant chacune un risque particulier: celui lié à la maintenance et aux mouvements d'armes ou parties d'armes nucléaires et celui afférent aux opérations de soutien des chaufferies nucléaires embarquées (Laroche et al., 2001). Les installations de l'île Longue permettent, en outre, de gérer quotidiennement la présence simultanée de deux à quatre bâtiments à propulsion nucléaire. À ces risques spécifiques s'ajoutent les accidents tels que les incendies importants dans l'environnement des installations sensibles, les pertes d'alimentation en énergie et les mouvements d'armes et de munitions conventionnelles.

À chaque type d'installation correspond un plan d'urgence adapté aux risques rencontrés, aussi les interventions sont-elles assurées en premier lieu par les moyens propres des spécialistes présents dans l'installation au moment de l'accident. Les installations présentes à l'Île Longue ne disposent pas des moyens humains et matériels pour faire face, au delà des premières actions réflexes, à une situation d'urgence. Il appartient aux services militaires de la base, sous la responsabilité du commandant de l'île Longue, coordonnateur du site, d'apporter à ces installations le soutien nécessaire à la gestion de la crise. Ces dispositions et l'organisation d'intervention de la base sont décrites dans le plan d'urgence interne du site (PUI/Site).

\section{Moyens d'intervention}

\subsection{Moyens humains}

L'intervention, en cas d'accident majeur, peut prendre simultanément plusieurs aspects: médical, technologique, radiologique et classique (lutte contre les sinistres associés). 
TABLEAU I

Organisation sécurité de la base.

Organization of the security on the site.

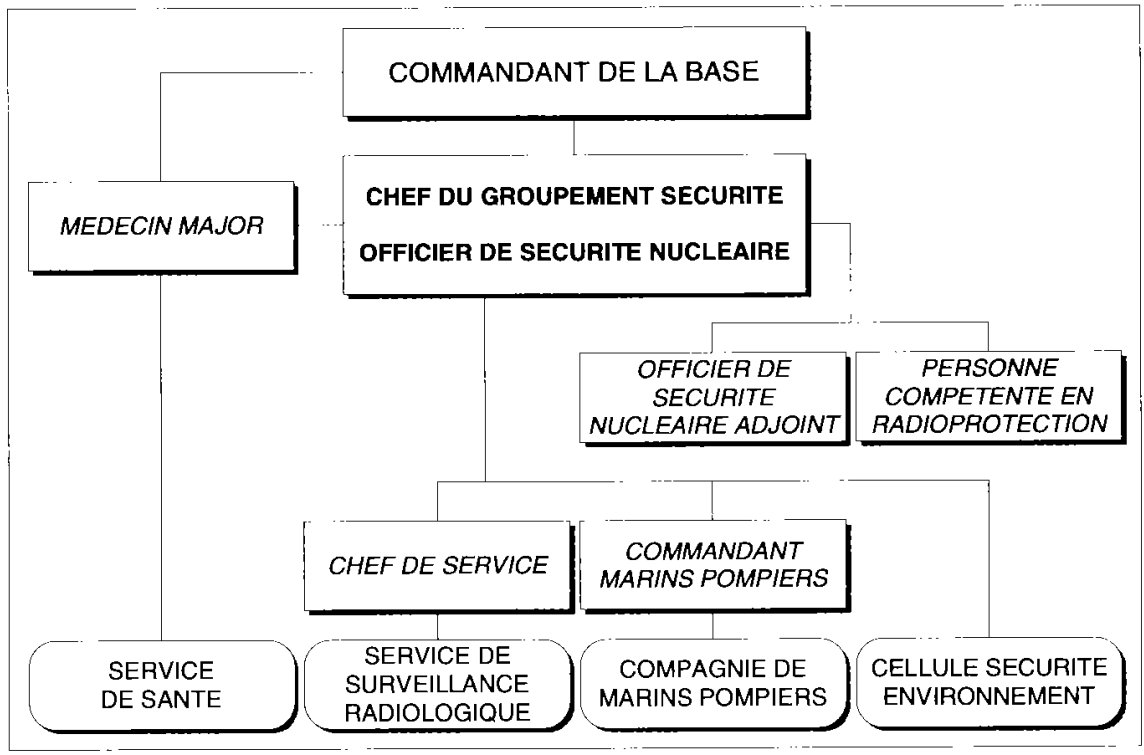

L'objectif global est de «durer» dans le temps et de mettre en cuvre des moyens suffisants afin de préserver le personnel de la base, les populations alentour, l'environnement et de rendre à l'installation accidentée un état de sûreté satisfaisant (IM 4900, 1993 ; IM 1830, 1994).

La base de l'île Longue dispose en propre de tous les services nécessaires pour assurer les multiples tâches d'intervention (Tab. I) :

- un service médical comprenant trois médecins épaulés par une équipe de douze infirmiers ;

- un service de surveillance radiologique, composé d'une trentaine de personnes majoritairement techniciens ou techniciens supérieurs en radioprotection ;

- une compagnie de marins pompiers, environ quatre-vingt personnes, dont une douzaine certifiés chefs de garde, qualification d'aptitude au rôle de COS (chef des opérations de secours);

- un service de soutien logistique spécialiste des travaux et actions réflexes dans l'environnement des sous-marins. 
Tous ces services assurent en heures non ouvrables une permanence dimensionnée pour assurer une première intervention dans l'attente du rappel du personnel d'astreinte. Les travaux présentant un risque particulier sont exécutés à l'exclusion de tout autre mouvement sensible et entraînent un renforcement significatif des équipes de permanence dans chacun des services d'intervention.

\subsection{Moyens matériels}

Les services sont équipés de moyens matériels leur permettant une large autonomie dans tous les aspects du traitement de la crise.

Le centre médical est structuré en trois parties principales (Laroche et al., 2002) :

- un centre de soins fonctionnant en permanence ;

- un poste d'accueil des blessés radiocontaminés (PABRC pour le traitement des urgences relatives, la décontamination fine et le traitement des contaminés comprenant une installation d'anthropogammamétrie. Le dispositif est complété par un mesureur de petits objets (MPO), un système de portiques de détection entrée/sortie et des balises de surveillance atmosphérique. Le personnel médical qui participe à l'intervention auprès des blessés fait l'objet d'une surveillance particulière. Il dispose de moyens dosimétriques individuels (actifs et passifs) et en cas de besoin il bénéficie de dispositifs atténuateurs de rayonnements, tabliers, lunettes et gants plombés (Berry et al., 1983) ;

- les éléments techniques modulaires (ETM), mis en œuvre pour le traitement des urgences absolues, sont composés d'un module bloc opératoire (Fig. 1) et d'un module réanimation avec quatre lits de soins intensifs (Fig. 2). Le module de chirurgie comprend une zone préparatoire près de son entrée et une zone opératoire. Son équipement est équivalent à celui d'un bloc moderne. Le module de réanimation est conçu pour la réanimation pré ou postopératoire de quatre blessés graves. Le poste d'accueil des blessés radiocontaminés associé aux éléments techniques modulaires forme le centre de traitement pour blessés radiocontaminés/Éléments techniques modulaires de l'île Longue (CTBRC/ETM).

Le service de surveillance radiologique dispose des appareils nécessaires aux mesures d'évaluation du risque radiologique dans le cadre de l'aide à la décision. À cette fin, le site est équipé d'un réseau de senseurs (2SNM : système de surveillance nucléaire de la marine) permettant de détecter un rejet important lors d'un accident, de remonter quantitativement et qualitativement au «terme source » et d'établir par des calculs de projection le danger encouru à court ou moyen terme par les populations environnantes. Prévenu, par convention d'information, le Préfet du département peut ainsi prendre préventivement toutes les mesures qu'ils jugera nécessaires pour la sauvegarde des populations dans le cadre du plan particulier d'intervention (PPI). 


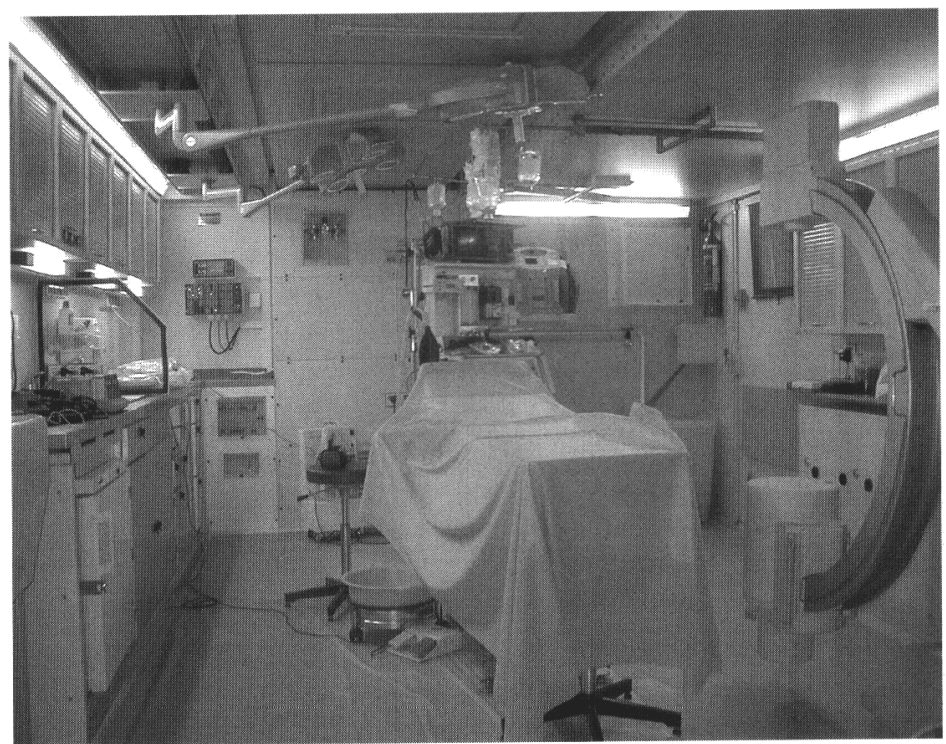

Figure 1-Module de chirurgie du CTBRC/ETM.

Surgery shelter of the CTBRC/ETM.

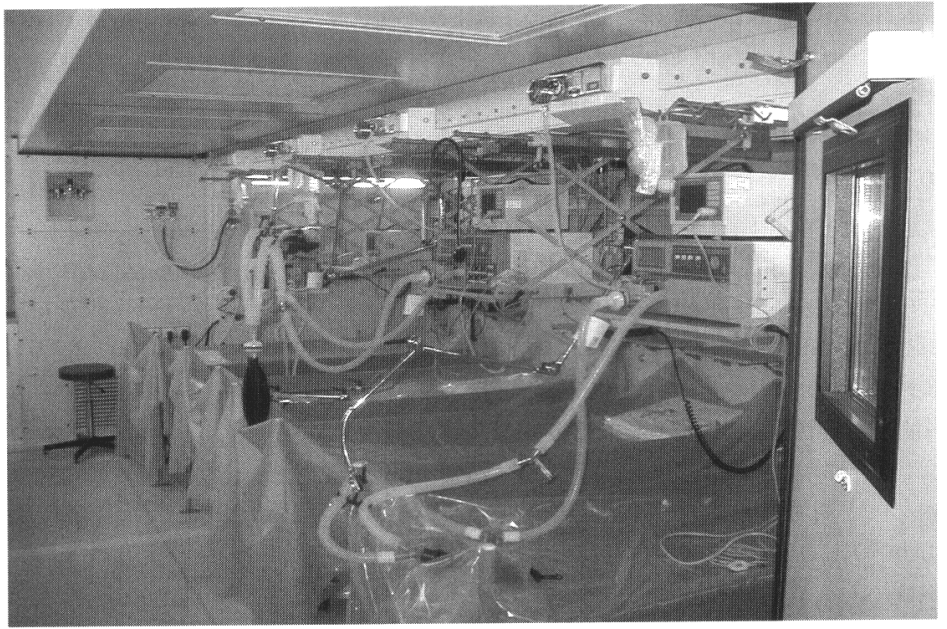

Figure 2 - Module de réanimation du CTBRC/ETM. Resuscitation shelter of the CTBRC/ETM. 
Le service dispose également des structures permettant le suivi, la décontamination et le contrôle du personnel tant intervenant qu'évacué des bâtiments et locaux refuge et qui sont dédiés exclusivement à cet usage.

La compagnie de marins pompiers est équipée d'une gamme complète de matériels moderne, véhicules et équipements, qui lui permettent d'intervenir simultanément pour le sauvetage de blessés et la lutte contre tout type de sinistre en ambiance radiologique et/ou chimique.

Ces trois principales composantes de l'intervention forment une unité géographique en zone «vie », zone à l'écart des risques industriels.

\section{Redéfinition du PUI/Site}

Le souci des responsables de l'intervention de l'île Longue a été d'optimiser l'utilisation de ces moyens humains et matériels en vue d'améliorer la rapidité et la qualité de l'intervention.

La réflexion a porté sur une meilleure intégration de ce personnel, de «culture » différente, au sein d'équipes d'intervention mixtes. L'effort s'est axé sur la transmission des informations, ordres et comptes rendus, aussi bien verticalement, des PC vers les intervenants, qu'horizontalement entre les différentes composantes.

La mise en place ou la modernisation des moyens d'intervention ont été orientées ces dernières années selon plusieurs axes de réflexion ou d'action prioritaires :

- optimisation de l'organisation de crise ;

- augmentation significative des effectifs ;

- plus grande adéquation des moyens d'intervention ;

- diminution du facteur « erreur humaine » par une meilleure formalisation des actions réflexes et une standardisation des modes d'intervention ;

- redéfinition des protocoles d'interface entre le coordonnateur de site et les directions responsables de la mise en œuvre des INBS ;

- implication des différents services pour le soutien des intervenants et du personnel évacué ;

- efforts sur la formation et l'entraînement.

\section{Organisation en phase initiale (Tab. II)}

Des permanences sont présentes à tous les niveaux :

- PZ (poste de zone) bassins pour l'installation chargée du soutien des chaufferies nucléaires embarquées ; 
TABLEAU II

Organisation en phase initiale.

Organization during initial stage.

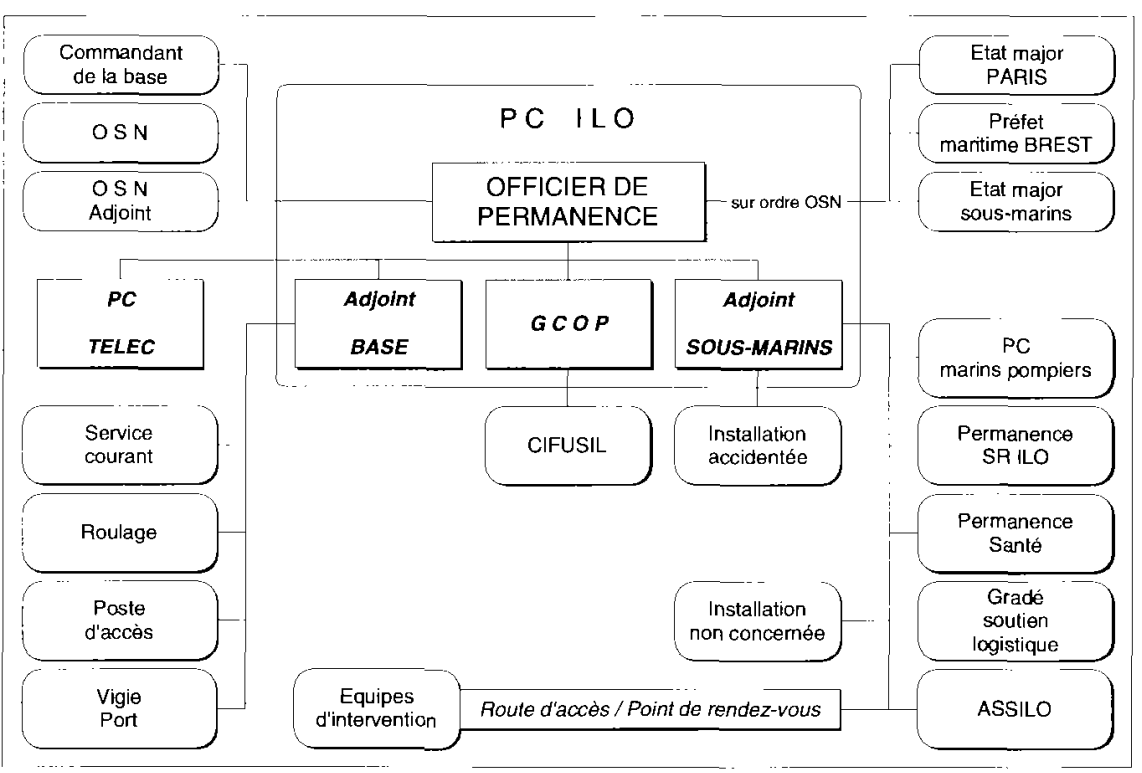

- PZ pyrotechnie pour l'installation nucléaire chargée de la maintenance, du montage et de l'entreposage des armes ou parties d'armes stratégiques;

- PC des marins pompiers ;

- Permanence du service de surveillance radiologique ;

- Médecin/infirmier de garde ;

- PC de l' ̂̂le Longue (PC ILO) assurant la permanence du commandement et la coordination des travaux sur le site (gestion des co-activités). 11 représente la structure centrale vers laquelle convergent toutes les informations et assure les fonctions de PC SECNUC puisqu'il dispose des paramètre des chaufferies embarquées et de la situation des autres installations (ateliers nucléaires et servitudes).

\subsection{Le PCILO}

Toute alarme, d'où qu'elle vienne, aboutit au PC ILO.

Le traitement de ces alarmes est alors effectué, à l'aide de fiches réflexes particularisées par le personnel présent. 
Le chef du PC, l'officier de permanence, est chargé de coordonner les actions de ses adjoints et de prévenir, selon l'estimation du degré de gravité, les différents responsables locaux, régionaux et nationaux.

Le premier de ses adjoint, l'adjoint sous-marins, est alors en communication avec l'installation accidentée par liaison directe. Il recueille les informations disponibles concernant le problème et la situation du système nucléaire militaire (SNM), les communique aux autres installations. Il met en alerte les premiers intervenants : le service de santé, les marins pompiers, le service de surveillance radiologique, le service de soutien logistique et, en cas d'accident impliquant l'armement nucléaire, les gendarmes du groupement de sécurité de l'armement nucléaire, chargé du contrôle gouvernemental. Il leur donne alors une route d'accès et un point de rendez-vous fonction des conditions météorologiques.

Le second adjoint, l'adjoint base, active les services n'ayant pas un rôle direct d'intervention dans l'installation :

- la gendarmerie maritime pour le maintien de l'ordre et la garde des accès ;

- l'intendance ;

- le service courant pour l'encadrement du personnel ;

- le roulage et le service portuaire pour les liaisons.

Le troisième adjoint active la compagnie des fusiliers marins qui assure le bouclage de la zone dite zone concernée par l'accident (ZCA).

Prévenus lors de la phase initiale, les responsables locaux rallient rapidement le PC ILO où l'officier de permanence leur présente la situation. Ils arment alors le poste de commandement d'intervention (PCI-ILO), contigu au PC ILO.

\section{Organisation en phase analyse / conduite (Tab. III)}

Cette phase est initialisée dès la décision du coordonnateur de site de mettre en œuvre le plan d'urgence interne du site.

\subsection{Le poste de commandement d'intervention (PCI-ILO)}

Le PCI-ILO est chargé d'assurer la direction de l'intervention sur le site dans les limites du périmètre de la base et de l'installation nucléaire.

Il centralise alors toutes les informations, à l'exception de la liaison technique médicale.

Il est dirigé par l'officier de sécurité nucléaire, directeur d'intervention, qui décide des moyens à engager, informe les autorités militaires, prend les mesures 


\section{TABLEAU III}

Organisation en phase stabilisation/développement.

Organization during stabilization /expansion stage.

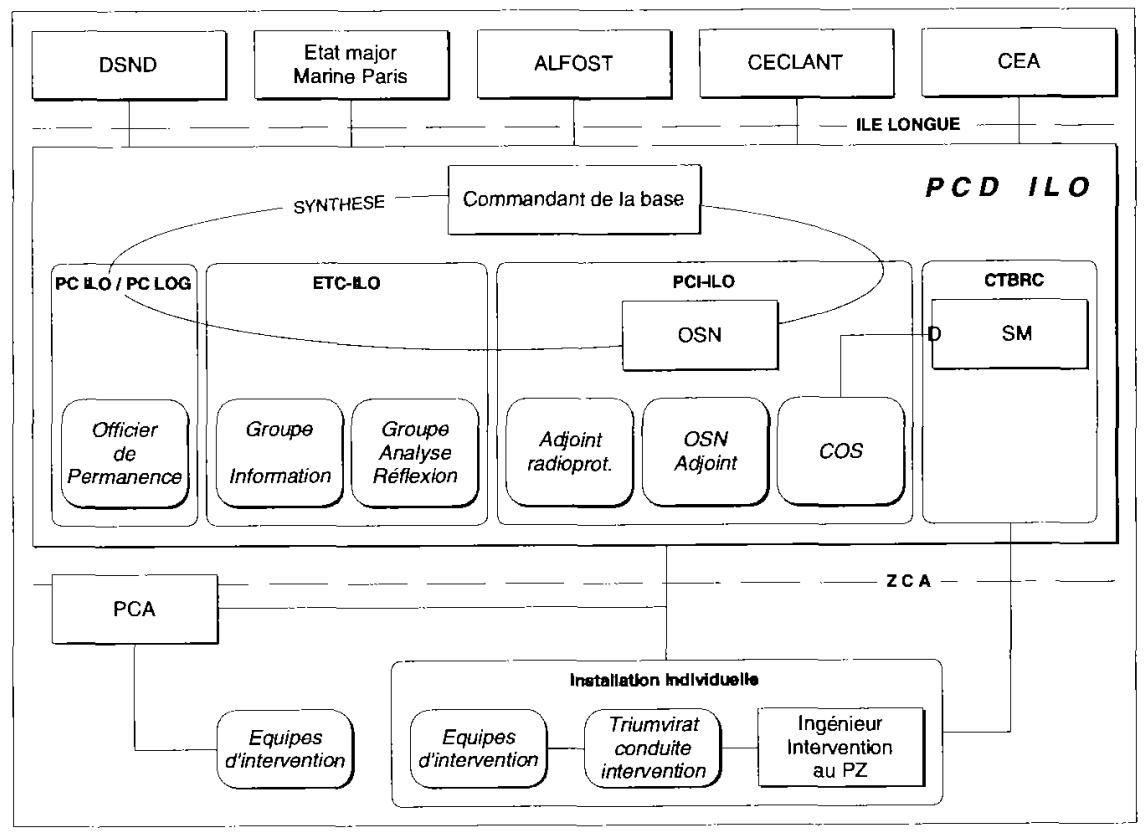

supplémentaires de protection du personnel et fournit aux responsables de mise en œuvre d'installation les moyens nécessaires à la gestion de l'accident.

L'officier de sécurité nucléaire adjoint assiste ou supplée l'OSN. Il assure la liaison entre l'OSN et les intervenants, le suivi technique de l'installation accidentée, le conseil au profit des responsables de mise en œuvre d'installation et veille à la continuité des servitudes de soutien des installations nucléaires.

Le commandant de la compagnie des marins pompiers arme un troisième poste : celui du chef des opérations de secours (COS).

Il dirige le secours aux blessés et la lutte contre les sinistres en liaison avec l'officier de garde marin pompier du jour, certifié « chef de garde », présent sur le lieu d'intervention.

Il assure la gestion des moyens en personnel et matériel nécessaires à l'intervention. 
Il coordonne la chaîne de traitement des blessés et radio-contaminés en liaison avec le médecin major de la base, directeur des secours médicaux (DSM).

Un quatrième poste est tenu par l'adjoint radioprotection (technicien supérieur en radioprotection), qui conseille l'OSN dans ce domaine. Assisté d'un technicien en radioprotection, il exploite le module environnement.

Un officier de police judiciaire de la gendarmerie maritime observe et note toutes les actions menées par l'autorité maritime dans le cadre de son intervention, à des fins d'enquête.

Si l'intervention s'installe dans la durée, un représentant du CEA/DAM en provenance d'un centre de cette direction, est intégré au PCI-ILO comme conseiller technique en intervention nucléaire (CTIN). Jusqu'à son arrivée, ce poste est assuré par un responsable du CEA/DAM du site.

\subsection{L'équipe technique de crise (ETC-ILO)}

L'équipe technique de crise agit au profit de l'installation accidentée et du coordonnateur de site en qualité de conseillère dans le cadre des actions à entreprendre afin de ramener l'installation nucléaire dans un état de sûreté acceptable. Elle est structurée en deux groupes.

\subsubsection{Le groupe analyse/réflexion}

Le groupe analyse/réflexion a pour missions de :

- rassembler, contrôler et gérer les informations concernant l'état de l'installation et son environnement ;

- analyser en temps réel la situation et son évolution ;

- rechercher auprès de l'assistance technique locale ou extérieure toute information utile à l'aide au diagnostic ; à cet effet, ce groupe est en liaison permanente avec la cellule de recueil de l'information marine (CRIMar) composée ellemême d'experts civils et militaires (CEA, Technicatome, STXN, ...) ;

- en fonction des données recueillies et du diagnostic effectué, proposer les mesures appropriées au chef d'installation.

\subsubsection{Le groupe information}

Le groupe information a pour missions :

- d'établir un bilan de la situation globale au profit de la cellule information du commandant de l'arrondissement maritime atlantique, de l'autorité militaire territoriale et de la CRIMar à l'état-major de la marine à Paris ; 
- de préparer le contenu des informations qui seront transmises aux médias via l'officier de relations publiques (ORP) puisque seul le commandant de la région maritime atlantique est habilité à informer, selon une convention, le préfet du département et les médias locaux. Ces communiqués sont soumis à l'approbation du coordonnateur de site ou de l'OSN par délégation.

\subsection{Le PCILO/PC LOG}

Après l'armement du PCI-ILO, il assure :

- la logistique nécessaire au ralliement du personnel rappelé ;

- la disponibilité et la fourniture des moyens logistiques nécessaires à l'intervention, hors moyens spécifiques ;

- le recensement du personnel confiné dans les bâtiments refuges.

\subsection{La cellule SYNTHÈSE}

Le coordonnateur de site provoque régulièrement ou sur ordre, l'établissement de la cellule synthèse composée des principaux responsables des structures de réflexion ou de décision mises en place.

Cette cellule permet d'harmoniser le fonctionnement des structures de gestion de la crise, de faire le point et de partager pour exploitation les informations de différentes origines.

\subsection{Le CTBRC/ETM}

Parallèlement et au même niveau que la cellule SECNUC, le médecin major de la base, directeur des secours médicaux (DSM), coordonne les missions du service médical, la mise en place et le fonctionnement de la chaîne médicale, du relevage jusqu'à l'évacuation des victimes.

Le CTBRC/ETM est divisé en trois chaînes distinctes : traitement des urgences absolues, traitement des urgences relatives et décontamination fine des personnes non blessées

Le médecin major de l'île Longue est le médecin chef du PABRC, il est officier traitant sur place pour l'entretien et la maintenance du dispositif, il assure l'instruction du personnel. Le médecin-chef des ETM est un anesthésisteréanimateur désigné par le médecin-chef de l'hôpital d'instruction des armées Clermont-Tonnerre. Il travaille en coordination avec le directeur des secours médicaux. L'équipe d'astreinte comprend deux chirurgiens, un anesthésiste, un radiologue, deux infirmiers anesthésistes, un infirmier de salle d'opération, 
trois infirmiers, un technicien matériel santé et un conducteur, soit douze personnes. Le PABRC est mis en service par le personnel médical et paramédical de l'île Longue. Le rappel du personnel serait adapté en fonction de la gravité de l'accident et du nombre de victimes.

\subsection{Structures subalternes}

L'OSN définit une zone concernée par l'accident (ZCA), comprenant la zone réellement contaminée, ou zone d'accident, entourée d'une zone propre dite « de sécurité ». Cette ZCA est immédiatement bouclée par les équipes de la compagnie de fusiliers marins de 1'île Longue.

\subsubsection{Le poste de commandement avancé (PCA)}

L'interface entre la ZCA et le reste de la base est assurée par le PCA. Ce point de passage obligé entre les deux zone, au vent du lieu d'accident, est dirigé par le chef du service de surveillance radiologique de la base.

Tout accès à la ZCA doit être alors justifié et enregistré.

Ce dispositif assure l'intervention en ZCA, hors installations nucléaires, la gestion dosimétrique du personnel intervenant, l'enregistrement et la mise en condition du personnel évacué des bâtiments refuges de la ZCA.

Le chef du PCA dispose d'un ensemble de moyens mobiles de transmission : ligne téléphonique dédiée, radios VHF/UHF et télécopieur.

Il dispose de véhicules ou structures mobiles spécialisées.

\subsubsection{Le poste médical avancé (PMA)}

Au PCA peut être associé un PMA. La décision d'implantation de celui-ci est décidée par le DSM en fonction du bilan sanitaire effectué par le médecin d'intervention.

Il permet la catégorisation, l'enregistrement, la surveillance médicale et la mise en condition des blessés évacués de la ZCA.

Cette structure est composée d'une tente gonflable où sont disposés huit brancards et d'une remorque spécialement équipée à cet effet contenant un lot de matériel médical complet. 


\section{TABLEAU IV}

Liaisons en intervention.

Contacts during intervention.

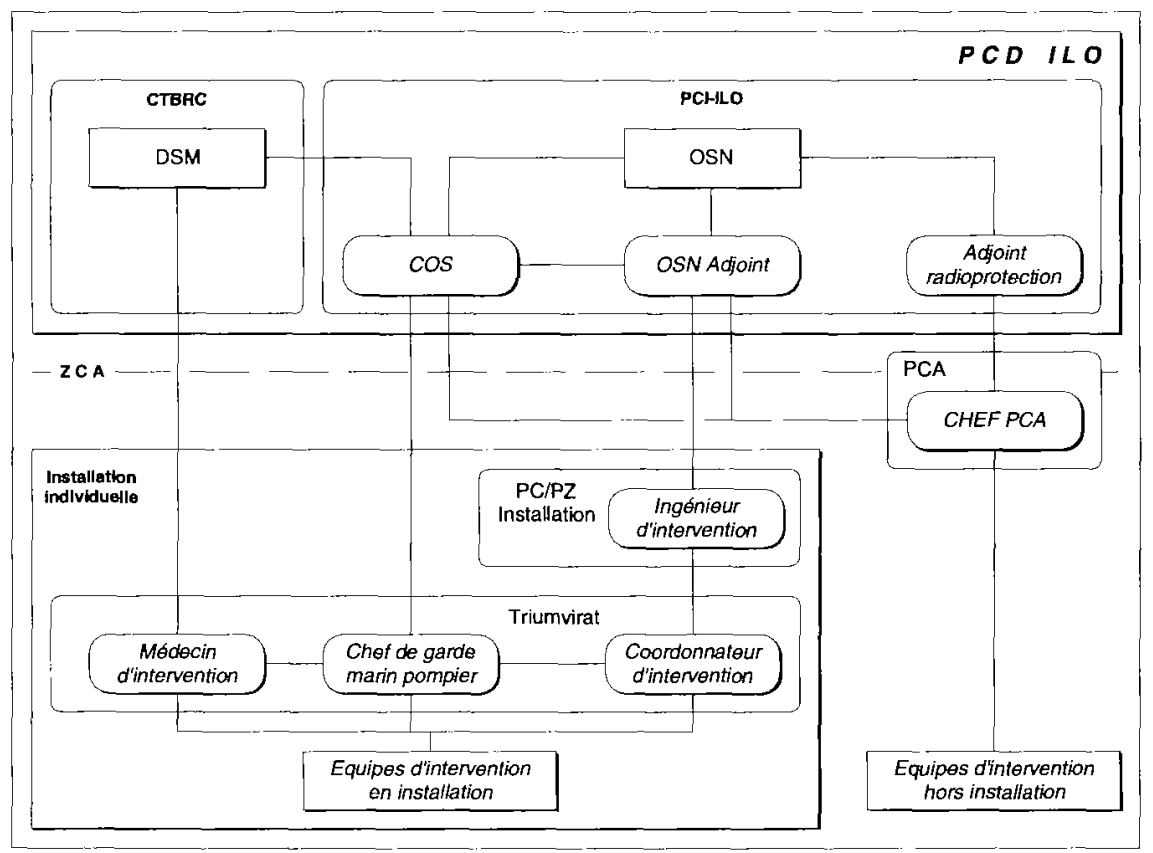

\subsection{Intervention dans les installations (Tab. IV)}

\subsubsection{Organisation}

L'organisation pour la gestion de l'intervention dans les installations, installations nucléaires de base secrètes ou bâtiments à propulsion nucléaire, est structurée selon les mêmes pôles que le PCI-ILO.

La responsabilité technique est assurée à partir de son poste de zone (PZ) par l'ingénieur d'intervention, en liaison directe avec l'officier de sécurité nucléaire adjoint au PCI-ILO.

Il est assisté par un «triumvirat » se tenant au plus près du point « zéro » de l'accident, en zone de soutien d'intervention (ZSI). Cette zone, au pire à faible risque, doit permettre d'appréhender les données physique du problème, 
de soutenir et orienter les actions d'intervention des équipes présentes et de communiquer et rendre compte avec les niveaux supérieurs.

Le triumvirat est composé :

- du coordonnateur d'intervention, expert technique appartenant au personnel de l'installation. Il est en liaison avec l'ingénieur d'intervention ;

- du médecin d'intervention qui communique ses diagnostics au DSM ;

- d'un officier de garde marin pompier certifié chef de garde chargé avec l'aide de ses équipes spécialisées du relevage des blessés et de la lutte contre les sinistres. Il est en contact avec le COS.

\subsubsection{Déroulement de l'intervention}

L'équipe de première intervention effectue le dégagement d'urgence des blessés des alentours du point « $0 »$, les place en zone de soutien d'intervention, repère en vue d'identification tous les blessés lors de la reconnaissance et traite un éventuel sinistre. Un technicien en radioprotection conseille l'équipe pendant la progression et effectue les prélèvements et mesures nécessaires à l'évaluation de la situation et du risque.

Le personnel d'intervention peut être placé en régime d'exposition d'urgence selon les modalités prévues par la réglementation.

L'ingénieur d'intervention est responsable de la gestion de l'intervention dans la limite de son installation, les actions à entreprendre étant définies dans le PUI de l'installation.

L'OSN reste responsable de la définition des équipements de protection individuels du personnel intervenant. Les missions demandées par l'exploitant sont effectuées conformément aux règles propres à chaque service intervenant.

Les intervenants, travaillant a priori sous appareil respiratoire isolant (ARI), parfois dans des locaux exigus (sous-marins), ne dispose que d'une autonomie limitée (environ 20 minutes pour du personnel entraîné). Les relèves nombreuses et rapprochées dans de bonnes conditions, ne sont possibles que grâce à la mise en place au PCA du module autonome de décontamination des intervenants.

L'intervention médicale commence parallèlement à la première intervention. Elle est exécutée par une équipe composée au minimum d'un médecin d'intervention et de l'équipe VSAB des marins pompiers (véhicule de secours aux asphyxiés et blessés) au niveau de la ZSI. 


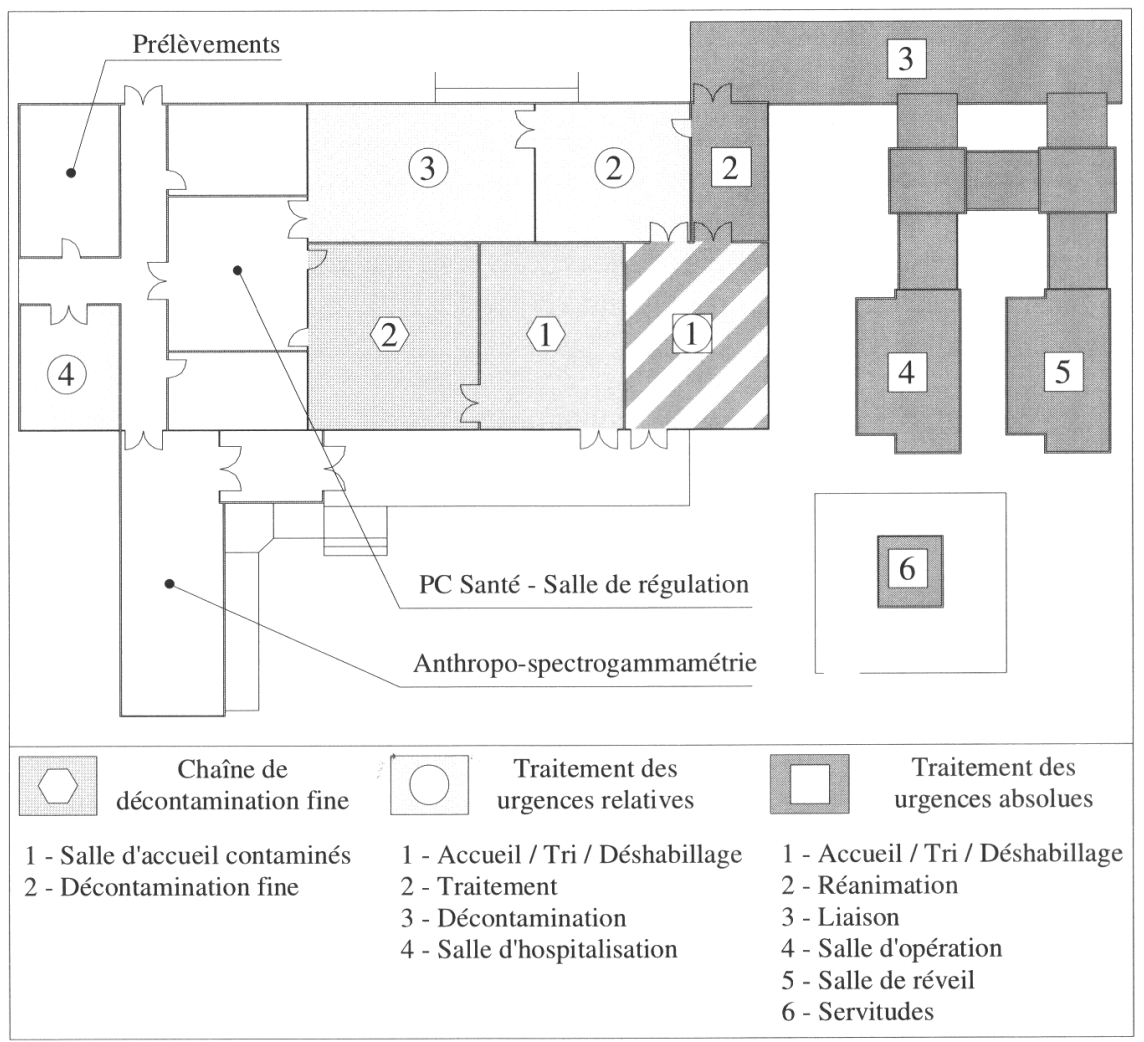

Figure 3-CTBRC/ETM.

CTBRC/ETM

Le médecin d'intervention, en liaison avec le DSM, procède à la catégorisation des blessés et réalise la médicalisation nécessaire à leur état. Le DSM définit alors l'ordre d'évacuation des blessés.

Les marins pompiers du VSAB conditionnent les blessés et les acheminent vers le PMA ou directement vers le CTBRC.

La prise en charge du personnel blessé est la priorité de toute intervention en cas d'accident à caractère radiologique.

Les urgences absolues (extrêmes urgences et premières urgences) sont transférées prioritairement vers le CTBRC (Fig. 3). 
Les urgences relatives, dont le traitement médico-chirurgical peut être différé sans risque pour le pronostic vital, sont acheminées en second lieu vers le CTBRC où elles seront décontaminées et soignées.

\subsubsection{Traitement des contaminés valides}

En temps normal, les contaminés valides, s'ils sont peu nombreux et en l'absence de blessés, sont acheminés directement vers le PABRC et passe par la chaîne de décontamination fine.

En cas d'afflux important de contaminés, ou en présence de blessés, ce personnel est traité en premier lieu au centre de tri et de décontamination sommaire (CTDS). Les contaminés résiduels, donc porteurs d'une contamination fixée, sont accompagnés et accueillis secondairement au PABRC où le personnel médical pratique une décontamination fine et traite la contamination interne supposée et éventuellement l'irradiation selon les protocoles établis.

Dans tous les cas, le cycle de traitement est achevé par les prélèvements biologiques et une anthropogammamétrie. Une cellule d'urgence médicopsychologique est prévue dans les locaux du centre médical. Cette cellule, qui a été testée et validée pendant les exercices, sera activée en cas d'accident à caractère catastrophique.

\subsection{Liaisons}

À l'exception de la liaison technique médicale entre le DSM, le médecin d'intervention et le PMA, les liaisons sont centralisées sur l'ensemble PCIILO/ETC-ILO afin d'éliminer toute communication parallèle et ainsi minimiser la perte d'information.

\subsection{Repli, protection et traitement du personnel des bâtiments refuges}

Le site de l'Île Longue est partagé en secteurs comprenant chacun un bâtiment refuge équipé de moyens de protection respiratoire individuels (masques) et de comprimés d'iodure de potassium. Les secteurs sont regroupés en zones supervisées par un $\mathrm{PC}$ de zone où se tient une permanence.

À la diffusion de l'alarme radiologique, le personnel, conformément aux panneaux de consignes d'évacuation de leur bâtiment et selon le fléchage mis en place, rallie le bâtiment refuge affecté à son secteur. 


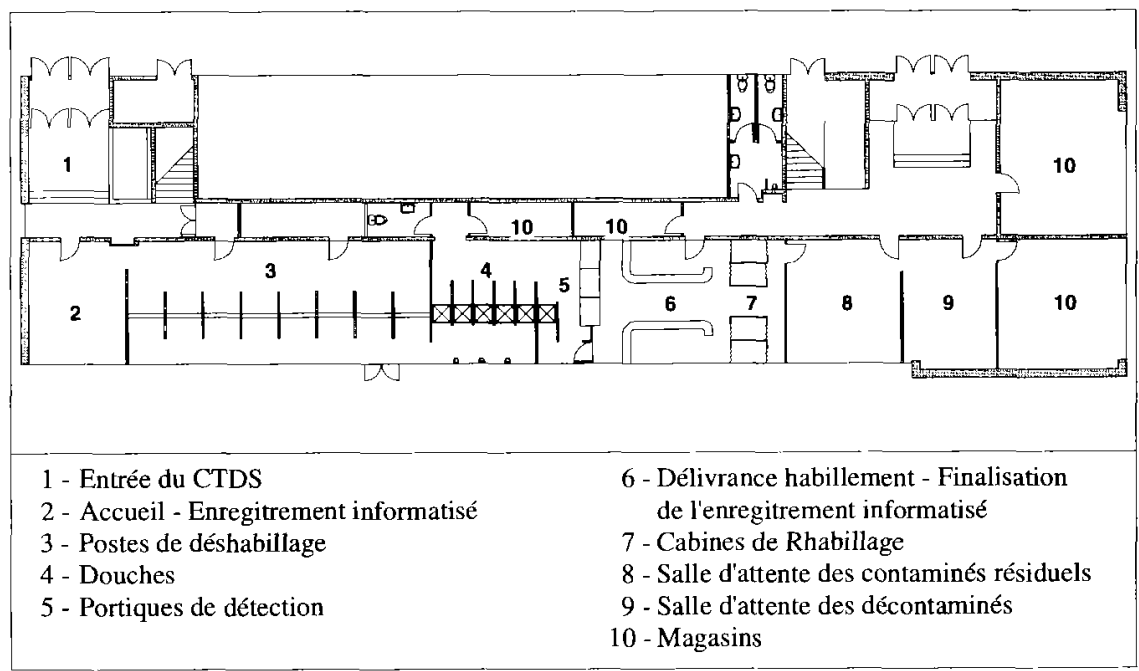

Figure 4-CTDS grande capacité.

Large capacity CTDS.

Le PC ILO, en liaison avec les PC de zone, assure le recensement du personnel des bâtiments refuges.

Le personnel du poste de commandement avancé est successivement chargé de deux missions :

- relever les noms du personnel réfugié et le numéro de téléphone des personnes à prévenir afin d'informer les familles au plus tôt ;

- procéder à l'évacuation progressive des bâtiments refuges, à l'enregistrement et au conditionnement de ce personnel.

Au PCA, ces personnes sont questionnées sur leur localisation et sur leur degré de protection individuelle au moment de l'accident. Ce questionnaire permet d'effectuer un premier tri et de traiter en priorité le personnel directement exposé.

Le personnel des bâtiments refuges situés hors des zones de retombées peut être contrôlé en sortie de bâtiment à l'aide de portiques mobiles mis en place par des équipe du service de surveillance radiologique.

Les évacués sont ensuite dirigés vers un CTDS de grande capacité (Fig. 4), environ cent personnes par heure, où il seront décontaminés et contrôlés dans l'attente de leur évacuation vers l'extérieur. Les contaminés résiduels sont pris en 
charge pour traitement de la contamination interne ou pour une décontamination fine au CTBRC.

\subsection{Pérennité de l'intervention}

Lorsque l'action est appelée à durer, les responsables font appel aux renforts des autres sites nucléaires des armées et de la marine en particulier. Les renforts du CEA sont systématiquement demandés. Les structures «vie» adaptées sont réservées à leur accueil sur le site.

\section{Formation - Entraînement}

La mise en forme de fiches réflexes et de réactions planifiées minimise la part du facteur humain, source d'erreurs en situation d'urgence, dans la gestion des premières interventions.

Ces dispositions ne sont pas suffisantes si la crise devait s'inscrire dans la durée. Aussi, un effort particulier a été apporté ces dernières années sur la formation et/ou l'information sur les risques présentés par les installations, la conduite à tenir et les conséquences en cas d'accident.

Tout le personnel de l'île Longue, soit environ deux milles personnes, est concerné par cette formation.

La formation se fait sur plusieurs plans:

- une formation élémentaire individuelle au niveau du service permettant la reconnaissance de la sirène d'alarme, la localisation des bâtiments refuges, la connaissance des consignes de bâtiments refuges et la sensibilisation aux risques radiologiques ;

- une formation du personnel assurant les fonctions de permanence qui a pour objectifs de connaître et comprendre l'organisation mise en place et de maîtriser les fiches réflexes associées à la fonction ;

- une formation aux fonctions d'intervenant pour la connaissance de l'organisation en intervention, des fiches réflexes et des fiches de tâche.

Les intervenants reçoivent une entraînement régulier dans le cadre d'exercices organisés au niveau local, régional ou national. Ceux-ci permettent de contrôler les connaissances acquises lors des séances de formation, de contrôler la disponibilité du matériel et d'améliorer par retour d'expérience, l'organisation mise en place.

La formation continue (hors école de formation) des intervenants non médicaux s'effectue au sein de chaque service, sur place ou à l'établissement 
technique de Bourges grâce aux instructeurs et moniteurs de l' ̂̂le Longue, ceux-ci ayant reçu une formation pédagogique adaptée. La formation des intervenants intègre aussi bien l'aspect théorique de base que l'aspect pratique par des travaux dirigés avec mises en situation.

L'instruction spécifique du personnel médical et paramédical pour la mise en cuvre du CTBRC/ETM est dispensée par le médecin major et le service de surveillance radiologique de l'île Longue. Cette instruction s'articule autour de quatre axes :

- le travail en ambiance contaminée ;

- la décontamination et la gestion des déchets contaminés ;

- l'activation des postes de travail sur le site ;

- la réalisation d'exercices en relation avec le PABRC.

\section{Retour d'expérience}

Le retour d'expérience, basé sur les exercices, montre que l'essentiel des dysfonctionnements provient des problèmes de communication, surtout à l'extérieur des installations, entre responsables et intervenants.

L'installation courant 2003 d'un nouveau système de transmissions UHF doit permettre de s'affranchir de ce type de difficultés.

\section{Conclusion}

L'organisation de l'intervention en cas d'accident à caractère radiologique sur le site de l'Île Longue possède une correspondance verticale entre les différents services intervenants et une grande structuration à tous les niveaux. Le principe de l'intervention combinée a été retenu et le partage équilibré des tâches ainsi que les facilités de communications horizontales sont privilégiées. Les actions sont standardisées quelle que soit l'installation concernée et quels que soient le jour et l'heure en raison de la présence permanente sur le site d'équipes d'intervention capables de travailler en autonomie. L'ensemble des acteurs de l'intervention bénéficie d'un effort d'équipement sans précédent ainsi que d'exercices et d'entraînements réguliers. L'organisation définie dans le plan d'urgence interne du site permet de faire face à toutes les situations accidentelles en complément des plans d'urgence interne propres à chaque installation. La priorité est donnée au traitement des blessés selon le principe suivant : l'urgence médico-chirurgicale prime sur l'urgence radiologique». Néanmoins, les missions de protection du personnel, des biens et de l'environnement sont assurées dans leur intégralité. 


\section{RÉFÉRENCES}

Berry J.-R., Mc Murray B.J., Jech J.J. (1983) 1976 Handford americium exposure incident : decontamination and treatment facility, Health Phys. 45(4), 883-892.

IM 4900 (1993) Instruction ministérielle $n^{\circ}$ 4900/DEF/CM.2/DR du 8 février 1993, relative à la sécurité nucléaire, modifiée du $\mathrm{l}^{\mathrm{er}}$ juin 1997.

IM 1830 (1994) Instruction ministérielle $n^{\circ}$ 1830/DEF/DCSSA/AST/TEC/I du 28 juillet 1994, relative à l'intervention du service de santé en cas d'accident à caractère radiologique en temps de paix, Bulletin Officiel de Armées $n^{\circ} 627$, Sécurité radiologique et protection contre les rayonnements ionisants, chapitre III, pp. 325-389.

Laroche P., Rousset J., Amabile J.-C., Roé H. (2001) Surveillance dosimétrique : résultats de équipages des forces sous-marines de 1989 à 1999, Radioprotection 36(3), 317-327.

Laroche P., Rousset J., Abiliou R., Roé H., Berthelot B., Lemaire L. (2002) Prise en charge des blessés radiocontaminés : une application originale des éléments techniques modulaires mise en œuvre à l'Île Longue, Médec. Armées 30(3), 275-282. 